\title{
Observations and Simulations of the Blue Straggler Star Radial Distribution: Clues on the Formation Mechanisms
}

\author{
Barbara Lanzoni ${ }^{1}$ \\ ${ }^{1}$ Dipartimento di Astronomia, Università di Bologna, \\ Via Ranzani 1, 40127 Bologna, Italy \\ email: barbara.lanzoni@bo.astro.it
}

\begin{abstract}
By means of high-resolution and wide-field observations in the UV and optical bands we have derived the radial distribution of the Blue Stragglers Star (BSS) population in a number of galactic globular clusters. Monte-Carlo dynamical simulations have then been used to interpret the observed radial distributions in terms of percentage of collisional and mass-transfer BSS populating each cluster. I will present the main results thus obtained and an overall cluster-tocluster comparison for the whole sample collected so far, mainly focusing on the clues that such an approach provides about the BSS formation mechanisms .
\end{abstract}

Keywords. globular clusters: general; stars: evolution; binaries: general; blue stragglers

\section{Blue Straggler Stars and their radial distribution}

In the color-magnitude diagrams (CMDs) of evolved stellar populations, like globular clusters (GCs), Blue straggler stars (BSS) populate a region which is brighter and bluer (hotter) than the Turn-Off (TO) point, along an extension of the Main Sequence. Thus, they appear as core hydrogen-burning objects, with masses larger than the normal cluster stars (Shara et al. 1997). The standard stellar evolution theory for single mass stars is unable to explain their existence, and a mechanism able to increase the initial stellar mass is required. Two main scenarios have been proposed for their formation: in the collisional scenario (Hills \& Day 1976), BSS are the end-products of stellar mergers induced by collisions (COL-BSS), while in the mass-transfer scenario (McCrea 1964; Zinn \& Searle 1976), BSS form by the mass-transfer activity between two companions in a binary system (MT-BSS), possibly up to the complete coalescence of the two stars. Hence, understanding the origin of BSS provides valuable insight both on binary evolution processes, and on the complex interplay between dynamics and stellar evolution in dense stellar systems.

The two formation mechanisms are likely to be at work simultaneously in every GC (Ferraro et al. 1993, 1997), with efficiencies that probably depend on the local density (Fusi Pecci et al. 1992; Ferraro et al. 1999a, 2003; Bellazzini et al. 2002). In fact, since stellar collisions are most probable in high-density environments, COL-BSS are expected to be formed preferentially in the cluster cores, while MT-BSS should mainly populate the cluster peripheries, where primordial binaries can more easily evolve in isolation. Since many GCs present high stellar crowding in their centers and large extensions in the sky, a combination of high-resolution and wide-field observations is needed to properly sample both the core and the outskirt environments. Moreover, the relatively high effective temperatures $\left(T_{e} \sim 7000-8000 \mathrm{~K}\right)$ of BSS make the ultraviolet (UV) passbands ideal for studying the photometric properties of these stars (see the contribution 
by Ferraro \& Lanzoni in this volume). For all these reasons, we are using a combination of high-resolution and wide-field observations, in the UV and optical bands, in a coordinated project aimed at understanding the formation mechanisms of these puzzling stars.

By adopting such kind of strategy for the study of M3 (Ferraro et al. 1997), 47 Tucanae (Ferraro et al. 2004), and NGC 6752 (Sabbi et al. 2004), BSS have been found to be more centrally concentrated than normal cluster stars (like red giant branch or horizontal branch stars; hereafter RGB and HB, respectively), consistently with the fact that they are more massive and that mass segregation process is at work in GCs. More surprisingly, the BSS population in these clusters also shows a bimodal radial distribution, i.e., with respect to $\mathrm{HB}$ or $\mathrm{RGB}$ stars, the $\mathrm{BSS}$ fraction is peaked in the center, decreases to a minimum at intermediate radii, and rises again in the outskirts. Such a bimodal radial distribution possibly contains precious information on the BSS formation mechanisms: in fact, the central peak might be due to COL-BSS formed in the core by stellar collisions, or to MT-BSS sunk to the center because of mass segregation and dynamical friction effects; the external rising branch might be produced by COL-BSS formed in the core and then kicked off by dynamical interactions, or by MT-BSS deriving from the normal evolution of binary systems in the cluster outskirts.

In order to disentangle among all these possibilities, we are using a modified version of the Monte-Carlo code originally described by Sigurdsson \& Phinney (1995)) for simulating the BSS dynamical evolution within a background cluster, with the aim of reproducing the observed bimodal radial distributions. The background cluster is described as the multi-mass King model that best fits the observed projected density profile. Two populations of BSS are considered: all stars with (random) initial position $r_{i}$ comprised within the cluster core radius $\left(r_{i}<r_{c}\right)$ and with non-zero natal kick velocity are assumed to be COL-BSS; those with $r_{i} \gg r_{c}$ and zero kick velocity are MT-BSS. Their dynamical evolution within the cluster potential well is due to the effects of dynamical friction and distant encounters. By varying the initial percentage of the two kinds of BSS, different radial distributions within the background cluster are obtained at the end of the simulations. The one that best reproduces the observed radial distribution gives the relative amount of COL- and MT-BSS that most likely populate the cluster. By using these simulations, Mapelli et al. $(2004,2006)$ have shown that the external rising branch observed in M3, 47 Tuc and NGC 6752 cannot be due to COL-BSS generated in the core and kicked out by stellar interactions. Instead, the observed bimodality can be explained only by assuming that a non-negligible fraction $(\sim 40 \%)$ of the BSS population is made of MT-BSS. These results demonstrate that detailed studies of the BSS radial distribution within GCs are powerful tools for better understanding the BSS formation mechanisms, and the effects of dynamical interactions on the (otherwise normal) stellar evolution.

In the following I present recent results obtained for three further galactic GCs (namely M5, NGC 1904 and NGC 6388) studied with the strategy described above.

The case of M5 (Lanzoni et al. 2007a): We have combined HST (WFPC2 and ACS) observations of the cluster center, in the UV and optical bands, with wide-field optical observations from the ground (with ESO-WFI). The BSS sample has been selected primarily on the basis of the position of stars in the $\left(m_{255}, m_{255}-U\right)$ plane, adopting a limiting magnitude $m_{255}=18.35$ ( one magnitude brighter than the TO) in order to avoid incompleteness bias and the possible contamination from TO and sub-giant branch stars. Once selected in the UV CMD, the bulk of the BSS in common with the opticalHST sample has been used to define the selection box and the limiting magnitude in the $(B, B-V)$ plane. The resulting BSS population of M5 amounts to a total of 60 objects. 
One BSS lies beyond the cluster tidal radius and might represent a very interesting case of a BSS evaporating from the cluster because of dynamical interactions. By dividing the sampled area in 8 concentric annuli, we have studied the radial distribution of the fraction of BSS with respect to HB stars $\left(N_{\mathrm{BSS}} / N_{\mathrm{HB}}\right)$. It has been found to be bimodal: highly peaked within the cluster core radius, decreasing to a minimum at $r \simeq 10 r_{c}$, and rising again outward. Dynamical simulations like those previously discussed suggest that the majority of BSS in M5 are collisional, with a content of MT-BSS ranging between $20 \%$ and $40 \%$ of the overall population (see Fig.1).

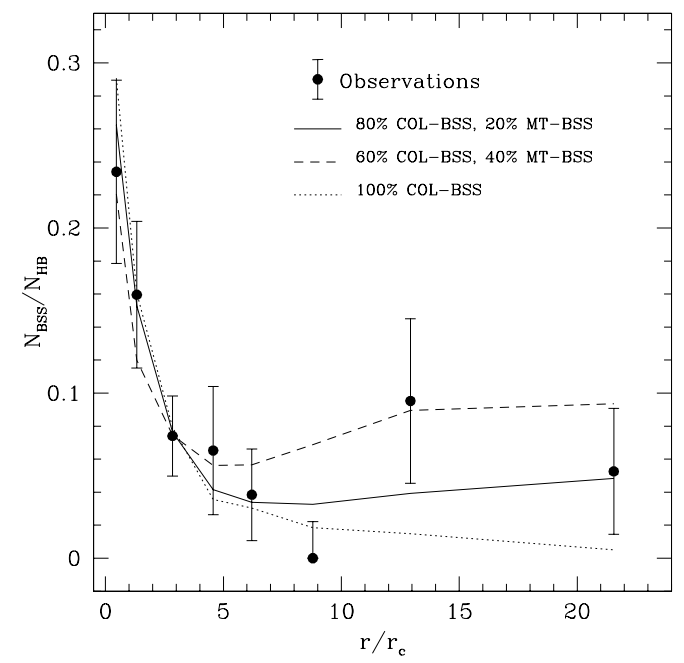

Figure 1. The BSS radial distribution of M5. The simulated distribution that best reproduces the observed one is shown as a solid line and is obtained by assuming $80 \%$ of COL-BSS and $20 \%$ of MT-BSS. The simulated distributions obtained by assuming $40 \%$ of MT-BSS (dashed line) and 100\% COL-BSS (dotted line) are also shown for comparison.

The case of NGC 1904 (Lanzoni et al. 2007b): We have studied the BSS population of NGC 1904, over the entire cluster extension, by combining high-resolution images obtained with HST-WFPC2, and wide-field observations, both from the ground, with ESO-WFI, and from the space, with GALEX. By adopting selection criteria similar to those discussed for M5, we have found a total of 39 BSS (with $m_{218} \leqslant 19.5$ and $V \leqslant 18.9$ ). Approximately $38 \%$ of the entire BSS population is found within the cluster core, while only $\sim 13 \%$ of HB stars are counted in the same region, thus indicating a significant overabundance of BSS in the center. The peak value is in good agreement with what is found in the case of M3, 47 Tucanae, NGC 6752 and M5 (Ferraro et al. 2004; Sabbi et al. 2004; Lanzoni et al. 2007a), but unlike these clusters, no significant upturn of the distribution at large radii has been detected in NGC 1904 (see second panel of Fig. 2). The Monte-Carlo simulations previously discussed suggest that only a negligible percentage $(0-10 \%)$ of MT-BSS is needed to reproduce the observed BSS radial distribution in this cluster.

The case of NGC 6388 (Dalessandro et al. 2007): To study the BSS population of NGC 6388, we have combined high-resolution images, in the UV and optical bands, obtained with HST (ACS and WFPC2) and wide-field observations from the ground with 


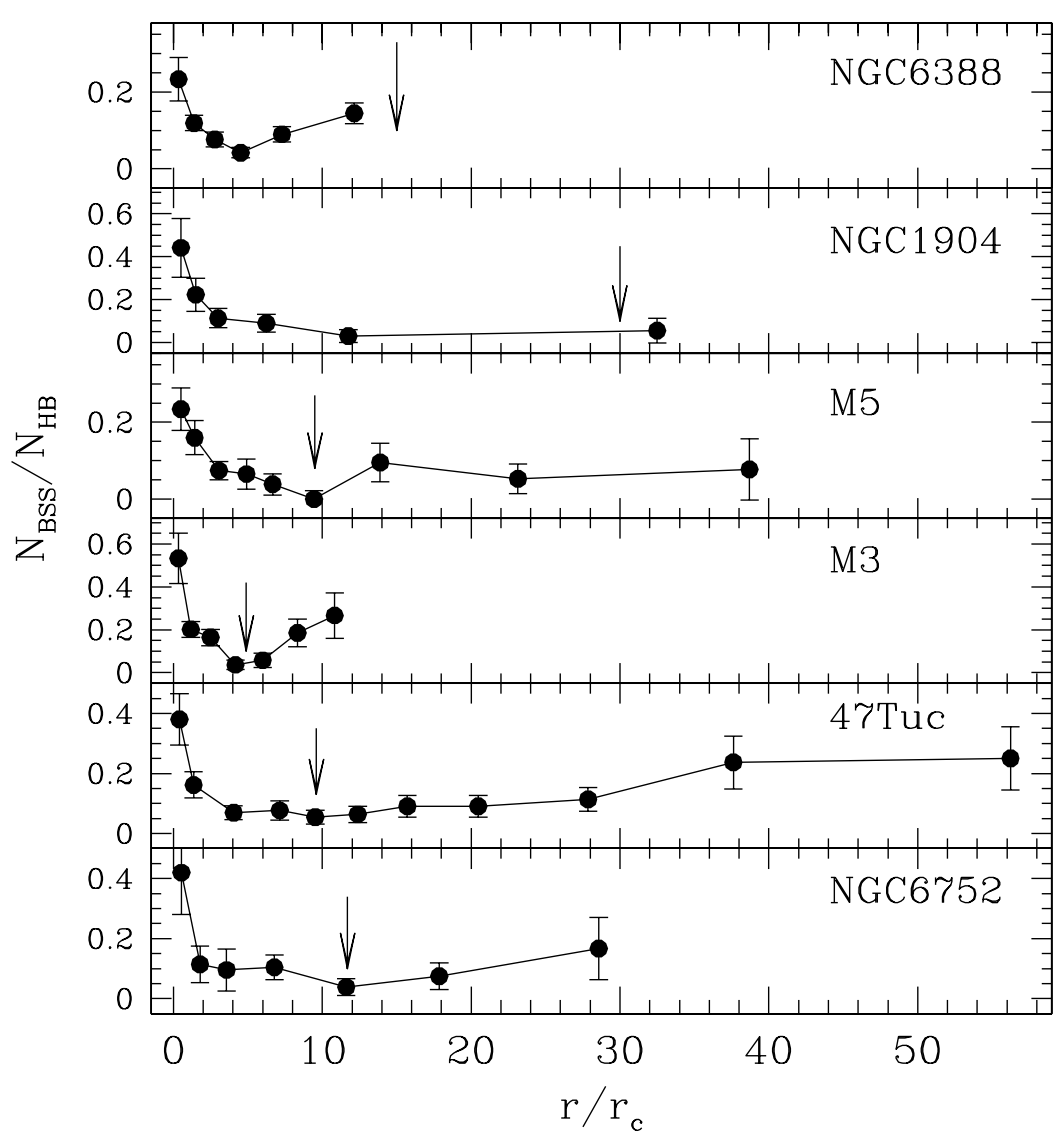

Figure 2. BSS radial distribution of NGC 1904, M5, M3, 47 Tucanae, and NGC 6752. The arrows indicate the position of the radius of avoidance of the clusters (see text), which nicely corresponds to the position of the minimum of the observed distributions, but in the case of NGC 6388 .

ESO-WFI. Because of the high degree of Galaxy star contamination, we have limited the analysis to the inner $110^{\prime \prime}$ from the cluster center, entirely covered by the HST data. As reference population representative of normal cluster stars, we have considered the HB red clump. The resulting BSS radial distribution is clearly bimodal, with a minimum at $r \simeq 5 r_{c}$ (see upper panel of Fig. 2). In order to infer the relative percentage of COLand MT-BSS in this cluster, we are currently performing the Monte-Carlo dynamical simulations discussed above.

\section{Discussion}

The BSS radial distributions of all the GCs observed to date with the strategy described above are compared in Fig.2.

In all cases BSS appear to be highly concentrated in the cluster centers, and in all but one (NGC 1904) they show a bimodal radial distribution. Dynamical simulations have demonstrated that the peripheral rising branch of the bimodal distributions cannot be generated by COL-BSS formed in the core and kicked out by stellar interactions. 
Instead, it can be properly reproduced only by assuming that a non-negligible fraction $(\sim 20-40 \%)$ of the BSS population is made of MT-BSS. The central peak is likely to be generated both by COL-BSS created inside the core by stellar collisions, and MT-BSS sunk to the center by mass segregation and dynamical friction processes.

These results seem to be confirmed by the fact that the observed position of the minimum nicely agrees with the estimated radius of avoidance of the systems (marked with arrows in Fig.2), i.e., the radius within which all the stars as massive as BSS (i.e., with $M \simeq 1.2 M_{\odot}$ ) are expected to have already sunk to the core due to dynamical friction effects. Up to date, the only exception is represented by NGC 6388, which shows a radius of avoidance about 3 times larger than the position of the observed minimum. Such a result is currently under deeper investigation, and might somehow be connected with other peculiarities shown by this GC, like the long blue tail of its HB (unexpected for such a high-metallicity cluster; Rich et al. 1997), the double sub-giant branch sequence (see the contribution by Piotto in this volume), and the possible presence of an intermediate-mass black hole in its center (Lanzoni et al. 2007c).

In conclusion, the study of the BSS radial distribution contain precious information on the formation mechanisms of these puzzling stars and the cluster evolution processes. Extending this kind of investigation to a larger sample of GCs with different structural and dynamical characteristics is required for identifying the cluster properties that mainly affect the BSS formation mechanisms and their relative efficiency.

High-resolution spectroscopic follow-up studies are also crucial for distinguishing between the two types of BSS. In fact, anomalous chemical abundances are expected at the surface of BSS resulting from MT activity (Sarna \& de Greve 1996), while they are not predicted in case of a collisional formation (Lombardi et al. 1995). The results found in the case of 47 Tucanae (Ferraro et al. 2006a; see also the contribution by Ferraro \& Lanzoni in this volume) are encouraging, and we are extending this kind of study to other galactic GCs.

\section{References}

Bellazzini, M., et al. 2002, AJ, 123, 1509

Dalessandro , E., et al. 2007, ApJ, submitted

Ferraro, F. R., et al. 1997, A\&A, 324, 915

Ferraro, F. R., Paltrinieri, B., Rood, R. T., \& Dorman, B. 1999a, ApJ, 522, 983

Ferraro, F. R., et al. 2003, ApJ, 588, 464

Ferraro, F. R., et al. 2004, ApJ, 603, 127

Ferraro, F. R., et al. 2006a, ApJ 647, L53

Fusi Pecci, F., et al. 1992, AJ, 104, 1831

Hills, J. G., \& Day, C. A. 1976, Astrophys. Lett., 17, 87

Lanzoni, B., et al. 2007a, ApJ, 663, 267

Lanzoni, B., et al. 2007b, ApJ 663, 1040

Lanzoni, B., et al. 2007c, ApJ 668, L139

Lombardi, J. C. Jr., Rasio, F. A., \& Shapiro, S. L. 1995, ApJ, 445, L117

Mapelli, M., et al. 2004, ApJ, 605, L29

Mapelli, M., et al. 2006, MNRAS, 373, 361

McCrea, W. H. 1964, MNRAS, 128, 147

Rich, R. M., et al. 1997, ApJ, 484, L25

Sabbi, E., Ferraro, F. R., Sills, A., \& Rood, R. T. 2004, ApJ, 617, 1296

Sarna, M. J., \& de Greve, J. P. 1996, QJRAS, 37, 11

Shara, M. M., Saffer, R. A., \& Livio, M. 1997, ApJ, 489, L59

Sigurdsson, S., \& Phinney, E. S. 1995, ApJS 99, 609

Zinn, R., \& Searle, L. 1976, ApJ, 209, 734 